\title{
On a Separatrix in the Gravitational Collapse to an Overcritical Electromagnetic Black Hole
}

\author{
Remo Ruffini $^{\text {a }}$, Luca Vitagliano ${ }^{\text {a }}$, She-Sheng Xue ${ }^{\text {a }}$ \\ a ICRA and Physics Department, University of Rome "La Sapienza", 00185 Rome, \\ Italy
}

\begin{abstract}
The dynamical properties of an electron-positron-photon plasma created by the vacuum polarization process occurring around a charged gravitationally collapsing core of an initially neutral star are examined within the framework of General Relativity and Quantum Field Theory. The Reissner-Nordström geometry is assumed to apply between the collapsing core and the oppositely charged remnant of the star. The appearance of a separatrix at radius $\bar{R}$, well outside the asymptotic approach to the horizon, is evidenced. The neutral electron-positron-photon plasma created at radii $r>\bar{R}$ self-propels outwards to infinity, following the classical PEMpulse analysis $[1,2]$. The plasma created at $r<\bar{R}$ remains trapped and follows the gravitational collapse of the core only contributing to the reduction of the electromagnetic energy of the black hole and to the increase of its irreducible mass. This phenomenon has consequences for the observational properties of gamma-ray bursts and is especially relevant for the theoretical prediction of the temporal and spectral structure of the short bursts.
\end{abstract}

Key words: EMBH, electron-positron plasma, gravitational collapse, gamma-ray bursts

PACS: 95.30.Lz, 97.60.Lf, 98.70.Rz

Preprint submitted to Elsevier Science

9 November 2018 
The formulation of the physics of the dyadosphere of an electromagnetic black hole (EMBH) has been until now approached by assuming the vacuum polarization process à là Sauter-Heisenberg-Euler-Schwinger $[3,4,5]$ in the field of an already formed Kerr-Newmann [6] or Reissner-Nordström black hole [7,8]. This acausal approach is certainly valid in order to describe the overall energetics and the time development of the gamma-ray bursts (GRBs) reaching a remarkable agreement between the observations and the theoretical prediction, in particular with respect to: a) the existence of a proper gamma-ray burst (P-GRB) [9], b) the afterglow detailed luminosity function and spectral properties $[10,11,12]$ and c) the relative intensity of the P-GRB to the afterglow $[13,10,11]$.

This acausal approach has to be improved by taking into account the causal dynamical process of the formation of the dyadosphere as soon as the detailed description on timescales of $10^{-4}-10^{-3}$ s of the $\mathrm{P}-\mathrm{GRB}$ are considered. Such a description leads to theoretical predictions on the time variability of the P-GRB spectra which may become soon testable by a new class of specially conceived space missions.

It is the aim of this letter to report progress in this theoretically challenging process which is marked by distinctive and precise quantum and general relativistic effects. These new results have been made possible by the recent progress in Refs. [14], [8] and especially [15]. There it was demonstrated the intrinsic stability of the gravitational amplification of the electromagnetic field

Email addresses: ruffini@icra.it (Remo Ruffini), vitagliano@icra.it (Luca Vitagliano), xue@icra.it (She-Sheng Xue). 
at the surface of a charged star core collapsing to an EMBH. The $e^{+} e^{-}$plasma generated by the vacuum polarization process around the core is entangled in the electromagnetic field [16]. The $e^{+} e^{-}$pairs do thermalize in an electronpositron-photon plasma on a time scale $10^{2}-10^{4}$ times larger than $\hbar / m_{e} c$ [15], where $c$ is the speed of light and $m_{e}$ the electron mass. As soon as the thermalization has occurred, a dynamical phase of this electrically neutral plasma starts following the considerations already discussed in $[1,2]$. While the temporal evolution of the $e^{+} e^{-} \gamma$ plasma takes place, the gravitationally collapsing core moves inwards, giving rise to a further amplified supercritical field, which in turn generates a larger amount of $e^{+} e^{-}$pairs leading to a yet higher temperature in the newly formed $e^{+} e^{-} \gamma$ plasma. We report, in the following, progress in the understanding of this crucial dynamical process: the main difference from the previous treatments is the fact that we do not consider an already formed EMBH but we follow the dynamical phase of the formation of dyadosphere and of the asymptotic approach to the horizon by examining the time varying process at the surface of the gravitationally collapsing core.

The space-time external to the surface of the spherically symmetric collapsing core is described by the Reissner-Nordström geometry [17] with line element

$$
d s^{2}=-\alpha^{2} d t^{2}+\alpha^{-2} d r^{2}+r^{2} d \Omega^{2}
$$

with $d \Omega^{2}=d \theta^{2}+\sin ^{2} \theta d \phi^{2}, \alpha^{2}=\alpha^{2}(r)=1-2 M / r+Q^{2} / r^{2}$, where $M$ and $Q$ are the total energy and charge of the core as measured at infinity. On the core surface, which at the time $t_{0}$ has radial coordinate $r_{0}$, the electromagnetic field strength is $\mathcal{E}=\mathcal{E}\left(r_{0}\right)=Q / r_{0}^{2}$. The equation of core's collapse is (see [14]):

$$
\frac{d r_{0}}{d t_{0}}=-\frac{\alpha^{2}\left(r_{0}\right)}{H\left(r_{0}\right)} \sqrt{H^{2}\left(r_{0}\right)-\alpha^{2}\left(r_{0}\right)}
$$


where $H\left(r_{0}\right)=\frac{M}{M_{0}}-\frac{M_{0}^{2}+Q^{2}}{2 M_{0} r_{0}}$ and $M_{0}$ is the core rest mass. Analytic expressions for the solution of Eq. (2) were given in [14]. We here recall that the dyadosphere radius is defined by $\mathcal{E}\left(r_{\mathrm{ds}}\right)=\mathcal{E}_{\mathrm{c}}=m_{e}^{2} c^{3} / e \hbar[7]$ as $r_{\mathrm{ds}}=\sqrt{e Q \hbar / m_{e}^{2} c^{3}}$, where $e$ is the electron charge. In the following we assume that the dyadosphere starts to be formed at the instant $t_{\mathrm{ds}}=t_{0}\left(r_{\mathrm{ds}}\right)=0$.

Having formulated the core collapse in General Relativity in Eq. (2), in order to describe the quantum phenomena, we consider, at each value of $r_{0}$ and $t_{0}$, a slab of constant coordinate thickness $\Delta r$ small in comparison with $r_{\mathrm{ds}}$ and larger than $\hbar / m_{e} c^{2}$. All the results will be shown to be independent on the choice of the value of $\Delta r$. In each slab the process of vacuum polarization leading to $e^{+} e^{-}$pair creation is considered. As shown in $[15,16]$ the pairs created oscillate $[18,19,20,21]$ with ultrarelativistic velocities and partially annihilate into photons; the electric field oscillates around zero and the amplitude of such oscillations decreases with a characteristic time of the order of $10^{2}-10^{4}$ $\hbar / m_{e} c^{2}$. The electric field is effectively screened to the critical value $\mathcal{E}_{\mathrm{c}}$ and the pairs thermalize to an $e^{+} e^{-} \gamma$ plasma. While the average of the electric field $\mathcal{E}$ over one oscillation is 0 , the average of $\mathcal{E}^{2}$ is of the order of $\mathcal{E}_{c}^{2}$, therefore the energy density in the pairs and photons, as a function of $r_{0}$, is given by [8]

$$
\epsilon_{0}\left(r_{0}\right)=\frac{1}{8 \pi}\left[\mathcal{E}^{2}\left(r_{0}\right)-\mathcal{E}_{c}^{2}\right]=\frac{\mathcal{E}_{c}^{2}}{8 \pi}\left[\left(\frac{r_{\mathrm{ds}}}{r_{0}}\right)^{4}-1\right]
$$

For the number densities of $e^{+} e^{-}$pairs and photons at thermal equilibrium we have $n_{e^{+} e^{-}} \simeq n_{\gamma}$; correspondingly the equilibrium temperature $T_{0}$, which is clearly a function of $r_{0}$ and is different for each slab, is such that

$$
\epsilon\left(T_{0}\right) \equiv \epsilon_{\gamma}\left(T_{0}\right)+\epsilon_{e^{+}}\left(T_{0}\right)+\epsilon_{e^{-}}\left(T_{0}\right)=\epsilon_{0},
$$


with $\epsilon$ and $n$ given by Fermi (Bose) integrals (with zero chemical potential):

$$
\begin{aligned}
& \epsilon_{e^{+} e^{-}}\left(T_{0}\right)=\frac{2}{\pi^{2} \hbar^{3}} \int_{m_{e}}^{\infty} \frac{\left(E^{2}-m_{e}^{2}\right)^{1 / 2}}{\exp \left(E / k T_{0}\right)+1} E^{2} d E, \quad \epsilon_{\gamma}\left(T_{0}\right)=\frac{\pi^{2}}{15 \hbar^{3}}\left(k T_{0}\right)^{4}, \\
& n_{e^{+} e^{-}}\left(T_{0}\right)=\frac{1}{\pi^{2} \hbar^{3}} \int_{m_{e}}^{\infty} \frac{\left(E^{2}-m_{e}^{2}\right)^{1 / 2}}{\exp \left(E / k T_{0}\right)+1} E d E, \quad n_{\gamma}\left(T_{0}\right)=\frac{2 \zeta(3)}{\hbar^{3}}\left(k T_{0}\right)^{3},
\end{aligned}
$$

where $k$ is the Boltzmann constantFrom the conditions set by Eqs. (4), (5), (6), we can now turn to the dynamical evolution of the $e^{+} e^{-} \gamma$ plasma in each slab. We use the covariant conservation of energy momentum and the rate equation for the number of pairs in the Reissner-Nordström geometry external to the star core:

$$
\nabla_{a} T^{a b}=0, \quad \nabla_{a}\left(n_{e^{+} e^{-}} u^{a}\right)=\overline{\sigma v}\left[n_{e^{+} e^{-}}^{2}(T)-n_{e^{+} e^{-}}^{2}\right]
$$

where $T^{a b}=(\epsilon+p) u^{a} u^{b}+p g^{a b}$ is the energy-momentum tensor of the plasma with proper energy density $\epsilon$ and proper pressure $p, u^{a}$ is the fluid 4-velocity, $n_{e^{+} e^{-}}$is the number of pairs, $n_{e^{+} e^{-}}(T)$ is the equilibrium number of pairs and $\overline{\sigma v}$ is the mean of the product of the $e^{+} e^{-}$annihilation cross-section and the thermal velocity of pairs. We follow closely the treatment which we developed for the consideration of a plasma generated in the dyadosphere of an already formed EMBH $[1,2]$. It was shown in $[1,2]$ that the plasma expands as a pairelectromagnetic pulse (PEM pulse) of constant thickness in the laboratory frame. Since the expansion, hydrodynamical timescale is much larger than the pair creation $\left(\hbar / m_{e} c^{2}\right)$ and the thermalization $\left(10^{2}-10^{4} \hbar / m_{e} c^{2}\right)$ time-scales, in each slab the plasma remains at thermal equilibrium in the initial phase of the expansion and the right hand side of the rate Eq. (7) is effectively 0, see Fig. 24 (second panel) of [11] for details.

If we denote by $\xi^{a}$ the static Killing vector field normalized at unity at spacial infinity and by $\left\{\Sigma_{t}\right\}_{t}$ the family of space-like hypersurfaces orthogonal to $\xi^{a}(t$ 
being the Killing time) in the Reissner-Nordström geometry, from Eqs. (7), the following integral conservation laws can be derived (see for instance [22,23])

$$
\int_{\Sigma_{t}} \xi_{a} T^{a b} d \Sigma_{b}=E, \quad \int_{\Sigma_{t}} n_{e^{+} e^{-}} u^{b} d \Sigma_{b}=N_{e^{+} e^{-}}
$$

where $d \Sigma_{b}=\alpha^{-2} \xi_{b} r^{2} \sin \theta d r d \theta d \phi$ is the vector surface element, $E$ the total energy and $N_{e^{+} e^{-}}$the total number of pairs which remain constant in each slab. We then have

$$
\left[(\epsilon+p) \gamma^{2}-p\right] r^{2}=\mathfrak{E}, \quad n_{e^{+} e^{-}} \gamma \alpha^{-1} r^{2}=\mathfrak{N}_{e^{+} e^{-}},
$$

where $\mathfrak{E}$ and $\mathfrak{N}_{e^{+} e^{-}}$are constants and

$$
\gamma \equiv \alpha^{-1} u^{a} \xi_{a}=\left[1-\alpha^{-4}\left(\frac{d r}{d t}\right)^{2}\right]^{-1 / 2}
$$

is the Lorentz $\gamma$ factor of the slab as measured by static observers. We can rewrite Eqs. (8) for each slab as

$$
\begin{aligned}
\left(\frac{d r}{d t}\right)^{2} & =\alpha^{4} f_{r_{0}}, \\
\left(\frac{r}{r_{0}}\right)^{2} & =\left(\frac{\epsilon+p}{\epsilon_{0}}\right)\left(\frac{n_{e^{+} e^{-0}}}{n_{e^{+} e^{-}}}\right)^{2}\left(\frac{\alpha}{\alpha_{0}}\right)^{2}-\frac{p}{\epsilon_{0}}\left(\frac{r}{r_{0}}\right)^{4}, \\
f_{r_{0}} & =1-\left(\frac{n_{e^{+}-}}{n_{e^{+} e^{-}-}}\right)^{2}\left(\frac{\alpha_{0}}{\alpha}\right)^{2}\left(\frac{r}{r_{0}}\right)^{4}
\end{aligned}
$$

where pedex ${ }_{0}$ refers to quantities evaluated at selected initial times $t_{0}>0$, having assumed $r\left(t_{0}\right)=r_{0}, d r /\left.d t\right|_{t=t_{0}}=0, T\left(t_{0}\right)=T_{0}$.

Eq. (11) is only meaningful when $f_{r_{0}}(r) \geq 0$. From the structural analysis of such equation it is clearly identifiable a critical radius $\bar{R}$ such that:

- for any slab initially located at $r_{0}>\bar{R}$ we have $f_{r_{0}}(r) \geq 0$ for any value of $r \geq r_{0}$ and $f_{r_{0}}(r)<0$ for $r \lesssim r_{0}$; therefore a slab initially located at a radial coordinate $r_{0}>\bar{R}$ moves outwards,

- for any slab initially located at $r_{0}<\bar{R}$ we have $f_{r_{0}}(r) \geq 0$ for any value of 
$r_{+}<r \leq r_{0}$ and $f_{r_{0}}(r)<0$ for $r \gtrsim r_{0}$; therefore a slab initially located at a radial coordinate $r_{0}<\bar{R}$ moves inwards and is trapped by the gravitational field of the collapsing core.

We define the surface $r=\bar{R}$, the dyadosphere trapping surface (DTS). The radius $\bar{R}$ of DTS is generally evaluated by the condition $\left.\frac{d f_{\bar{R}}}{d r}\right|_{r=\bar{R}}=0 . \bar{R}$ is so close to the horizon value $r_{+}$that the initial temperature $T_{0}$ satisfies $k T_{0} \gg m_{e} c^{2}$ and we can obtain for $\bar{R}$ an analytical expression. Namely the ultrarelativistic approximation of all Fermi integrals, Eqs. (5) and (6), is justified and we have $n_{e^{+} e^{-}}(T) \propto T^{3}$ and therefore $f_{r_{0}} \simeq 1-\left(T / T_{0}\right)^{6}\left(\alpha_{0} / \alpha\right)^{2}\left(r / r_{0}\right)^{4}(r \leq \bar{R})$. The defining equation of $\bar{R}$, together with (13), then gives

$$
\bar{R}=2 M\left[1+\left(1-3 Q^{2} / 4 M^{2}\right)^{1 / 2}\right]>r_{+}
$$

In the case of an EMBH with $M=20 M_{\odot}, Q=0.1 M$, we compute:

- the fraction of energy trapped in DTS:

$$
\bar{E}=\int_{r_{+}<r<\bar{R}} \alpha \epsilon_{0} d \Sigma \simeq 0.53 \int_{r_{+}<r<r_{\mathrm{ds}}} \alpha \epsilon_{0} d \Sigma
$$

- the world-lines of slabs of plasma for selected $r_{0}$ in the interval $\left(\bar{R}, r_{\mathrm{ds}}\right)$ (see Fig. 1);

- the world-lines of slabs of plasma for selected $r_{0}$ in the interval $\left(r_{+}, \bar{R}\right)$ (see Fig. 2).

At time $\bar{t} \equiv t_{0}(\bar{R})$ when the DTS is formed, the plasma extends over a region of space which is almost one order of magnitude larger than the dyadosphere and which we define as the effective dyadosphere. The values of the Lorentz $\gamma$ factor, the temperature and $e^{+} e^{-}$number density in the effective dyadosphere are given in Fig. 3 . 
In conclusion we see how the causal description of the dyadosphere formation can carry important messages on the time variability and spectral distribution of the P-GRB due to quantum effects as well as precise signature of General Relativity.

\section{References}

[1] R. Ruffini, J. D. Salmonson, J. R. Wilson, S.-S. Xue, A $\& A 350$ (1999) 334.

[2] R. Ruffini, J. D. Salmonson, J. R. Wilson, S.-S. Xue, A\&\&A 359 (2000) 855.

[3] F. Sauter, Zeits. Phys. 69 (1931) 742.

[4] W. Heisenberg, H. Euler, Zeits. Phys. 98 (1935) 714.

[5] J. Schwinger, Phys. Rev. 82 (1951) 664;

[6] T. Damour, R. Ruffini, Phys. Rev. Lett. 35 (1975) 463.

[7] G. Preparata, R. Ruffini, S.-S. Xue, $A \& A A 338$ (1998) L87.

[8] R. Ruffini, L. Vitagliano, Phys. Lett. B 545 (2002) 233.

[9] R. Ruffini, C. L. Bianco, P. Chardonnet, F. Fraschetti, S.-S. Xue, ApJ 555 (2001) L107.

[10] R. Ruffini, C. L. Bianco, P. Chardonnet, F. Fraschetti, S.-S. Xue, Int. J. Mod. Phys. D 12 (2003) 173.

[11] R. Ruffini, C. L. Bianco, P. Chardonnet, F. Fraschetti, L. Vitagliano, S.-S. Xue, Proceedings of the Xth Brazilian School of Cosmology and Gravitation, Eds. M. Novello, S. E. Perez Bergliaffa, AIP Conference Proceedings 668 (2003) 16.

[12] R. Ruffini, C. L. Bianco, P. Chardonnet, F. Fraschetti, S.-S. Xue, ApJ, submitted to. 
[13] R. Ruffini, C. L. Bianco, P. Chardonnet, F. Fraschetti, S.-S. Xue, ApJ 555 (2001) L113.

[14] C. Cherubini, R. Ruffini, L. Vitagliano, Phys. Lett. B 545 (2002) 226.

[15] R. Ruffini, L. Vitagliano, S.-S. Xue, Quantum Aspects of Beam Physics, 28th Advanced IFCA Beam Dynamics Workshop, Ed. Pisin Chen, (World Scientific, Singapore, 2003) in press; astro-ph/0304306.

[16] R. Ruffini, L. Vitagliano, S.-S. Xue, Phys. Lett. B 559 (2003) 12.

[17] A. Papapetrou, Lectures on General Relativity (D. Re idel publishing company, Dordrecht Boston, 1974).

[18] Y. Kluger, J. M. Eisenberg, B. Svetitsky, F. Cooper, E. Mottola, Phys. Rev. Lett. 67 (1991) 2427.

[19] Y. Kluger, J. M. Eisenberg, B. Svetitsky, F. Cooper, E. Mottola, Phys. Rev. D 45 (1992) 4659.

[20] F. Cooper, J. M. Eisenberg, Y. Kluger, E. Mottola, B. Svetitsky, Phys. Rev. D 48 (1993) 190.

[21] J. C. R. Bloch et al., Phys. Rev. D 60 (1999) 116011.

[22] W. G. Dixon, Rend. Scuola Intern. "Enrico Fermi" LXVII Course (Società italiana di Fisica, Bologna, 1979) 156.

[23] J. L. Synge, Relativity: the General Theory (North-Holland publishing company, Amsterdam, 1960). 


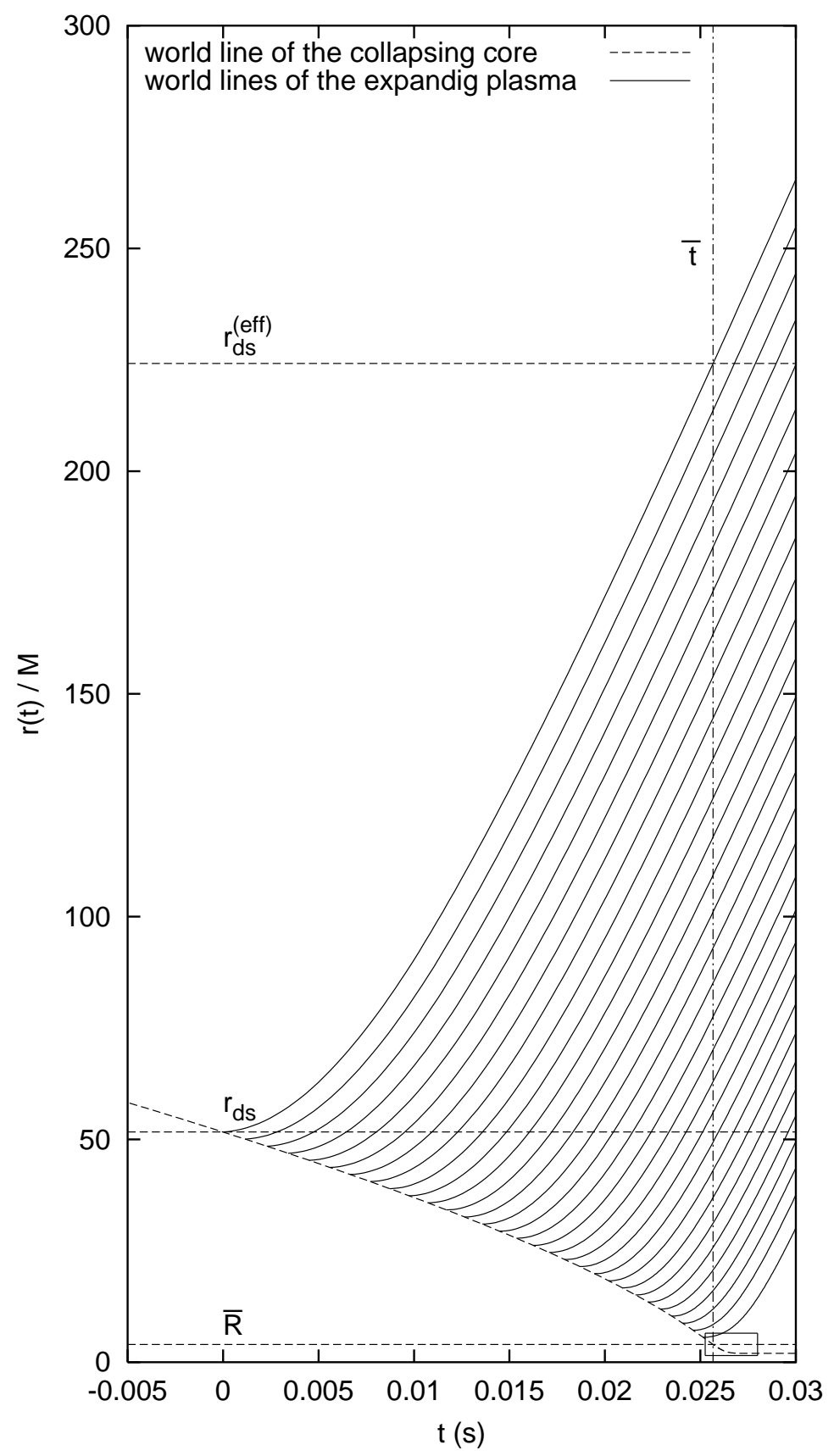

Fig. 1. World line of the collapsing charged core (dashed line) as derived from Eq. (2) for an EMBH with $M=20 M_{\odot}, Q=0.1 M$; world lines of slabs of plasma for selected radii $r_{0}$ in the interval $\left(\bar{R}, r_{\mathrm{ds}}\right)$. At time $\bar{t}$ the expanding plasma extends over a region which is almost one order of magnitude larger than the dyadosphere. The small rectangle in the right bottom is enlarged in Fig. 2. 


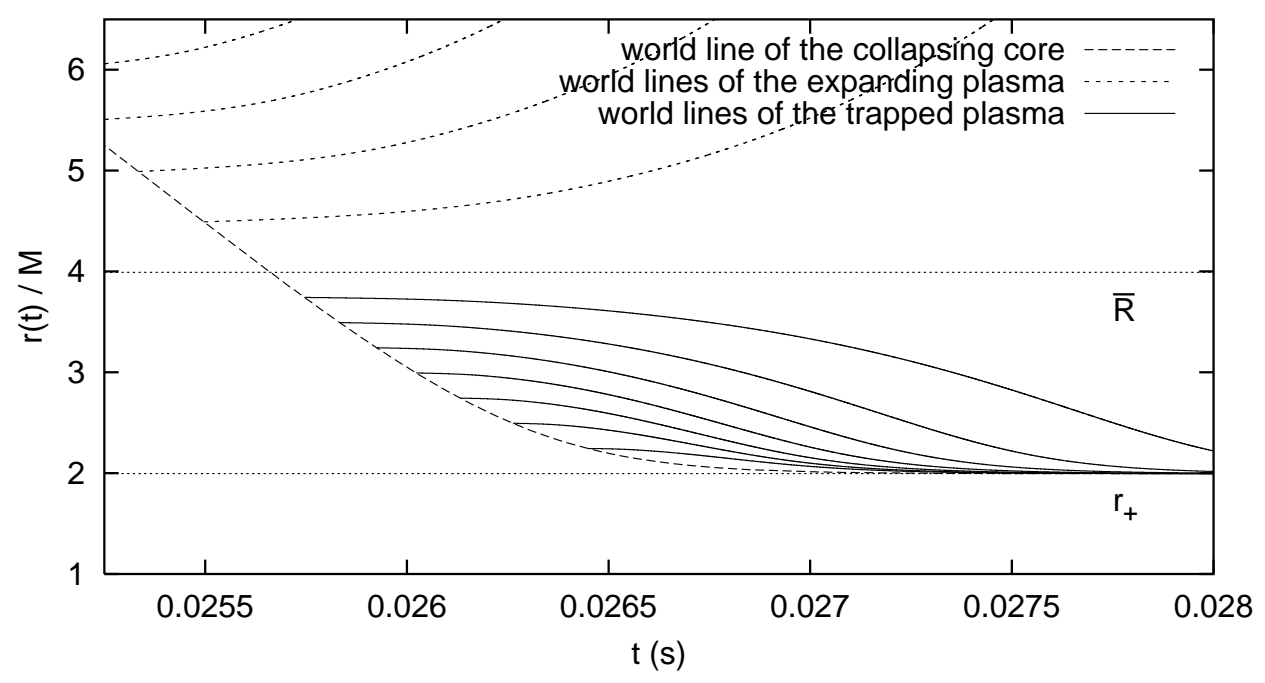

Fig. 2. Enlargement of the small rectangle in the right bottom of Fig. 1. World-lines of slabs of plasma for selected radii $r_{0}$ in the interval $\left(r_{+}, \bar{R}\right)$. 

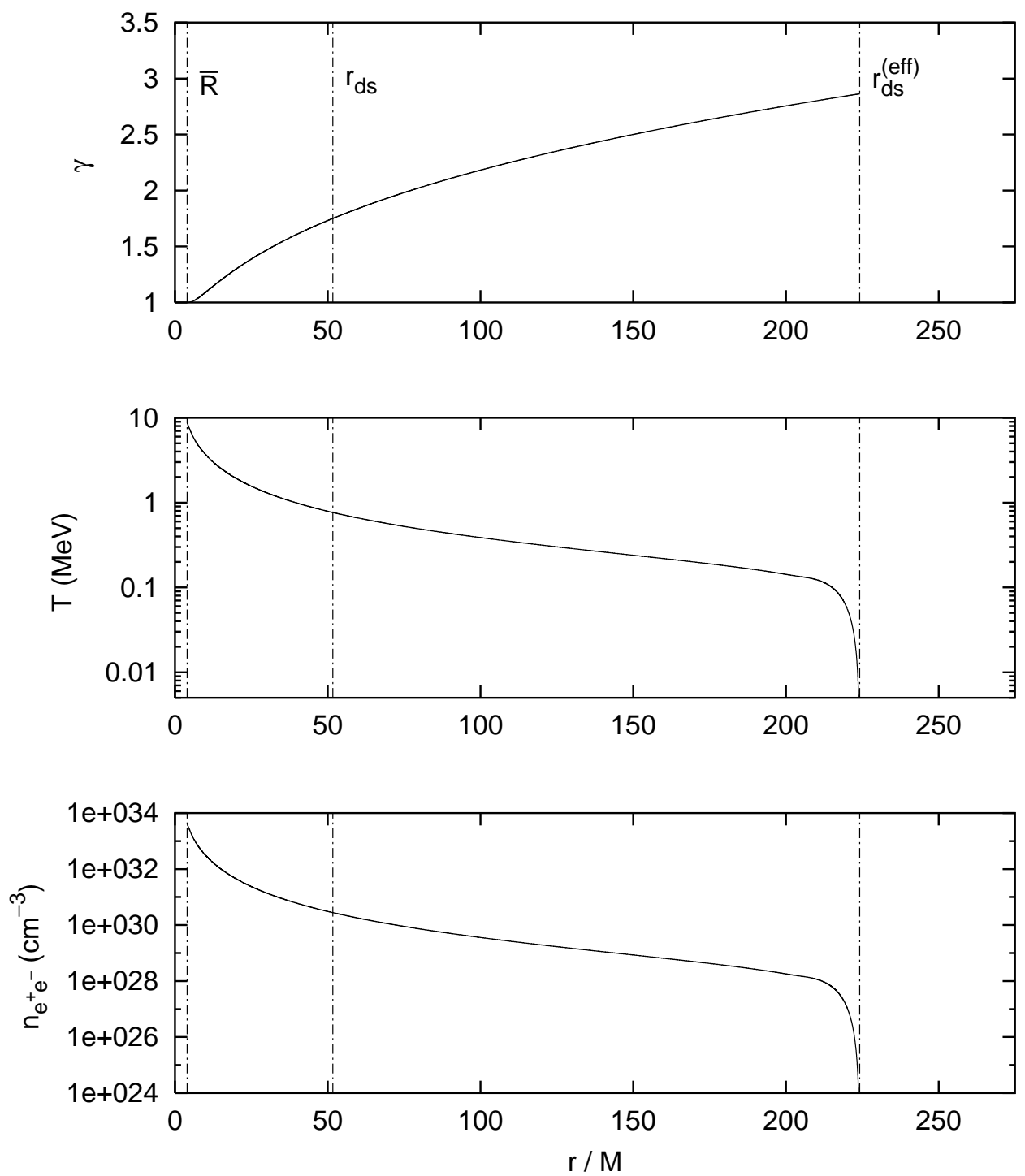

Fig. 3. Physical parameters in the effective dyadosphere: Lorentz $\gamma$ factor, proper temperature and proper $e^{+} e^{-}$number density as functions at time $\bar{t}$ for an EMBH with $M=20 M_{\odot}$ and $Q=0.1 M$. 